\title{
Téoros
}

Revue de recherche en tourisme

\section{Les stratégies de conversion touristique des stades d'eau vive olympiques}

\author{
Une approche par l'avantage métropolitain
}

\section{Antoine Marsac}

Volume 33, numéro 1, 2014

Méga-événements sportifs

URI : https://id.erudit.org/iderudit/1036720ar

DOI : https://doi.org/10.7202/1036720ar

Aller au sommaire du numéro

Éditeur(s)

Université du Québec à Montréal

ISSN

0712-8657 (imprimé)

1923-2705 (numérique)

Découvrir la revue

Citer cet article

Marsac, A. (2014). Les stratégies de conversion touristique des stades d'eau vive olympiques : une approche par l'avantage métropolitain. Téoros, 33(1), 67-74. https://doi.org/10.7202/1036720ar
Résumé de l'article

Cet article analyse l'implantation de sites olympiques - les stades d'eau vive (SEV) - comme un enjeu stratégique pour développer le tourisme urbain. Il s'agit d'étudier comment ces équipements conçus à l'origine pour le slalom, discipline apparue aux Jeux olympiques (JO) en 1972 puis réintroduite à partir de 1992, sont réutilisés pour l'accueil du public. J'analyserai les jeux d'acteurs autour de la reconversion de ces équipements en lieu touristique pour comprendre comment la délocalisation de ces sites a engendré une fréquentation des périphéries métropolitaines. L’objectif consiste à appréhender les phénomènes qui participent à leur mise en tourisme. Des études de cas (Yin, 2003) de quatre SEV des JO de Munich (1972), Sydney (2000), Athènes (2004) et Londres (2012) servent d'appui à la recherche. L'étude d'un corpus d'articles issus de la presse spécialisée complète le traitement d'archives du Comité international olympique (CIO) et de la Fédération internationale de canoë. Lors de la candidature des villes hôtes, les élus se servent des SEV pour accroître la notoriété des métropoles. Loin de s'aligner sur un modèle unique, l'examen des stratégies de développement territorial montre combien la question du devenir des installations olympiques reste tenace dans l'optique d'un avantage métropolitain (Halbert, 2009) vis-à-vis des sites délocalisés.
Ce document est protégé par la loi sur le droit d'auteur. L’utilisation des services d’Érudit (y compris la reproduction) est assujettie à sa politique d'utilisation que vous pouvez consulter en ligne.

https://apropos.erudit.org/fr/usagers/politique-dutilisation/ 


\title{
Les stratégies de conversion touristique des stades d'eau vive olympiques Une approche par l'avantage métropolitain
}

\author{
Antoine MARSAC, docteur \\ Maître de conférences \\ Laboratoire SPMS \\ Université de Bourgogne \\ antoine.marsac@u-bourgogne.fr
}

\begin{abstract}
RÉSUMÉ : Cet article analyse l'implantation de sites olympiques — les stades d'eau vive (SEV) — comme un enjeu stratégique pour développer le tourisme urbain. II s'agit d'étudier comment ces équipements conçus à l'origine pour le slalom, discipline apparue aux Jeux olympiques (J0) en 1972 puis réintroduite à partir de 1992, sont réutilisés pour l'accueil du public. J'analyserai les jeux d'acteurs autour de la reconversion de ces équipements en lieu touristique pour comprendre comment la délocalisation de ces sites a engendré une fréquentation des périphéries métropolitaines. L'objectif consiste à appréhender les phénomènes qui participent à leur mise en tourisme. Des études de cas (Yin, 2003) de quatre SEV des JO de Munich (1972), Sydney (2000), Athènes (2004) et Londres (2012) servent d'appui à la recherche. L'étude d'un corpus d'articles issus de la presse spécialisée complète le traitement d'archives du Comité international olympique (CIO) et de la Fédération internationale de canoë. Lors de la candidature des villes hôtes, les élus se servent des SEV pour accroître la notoriété des métropoles. Loin de s'aligner sur un modèle unique, l'examen des stratégies de développement territorial montre combien la question du devenir des installations olympiques reste tenace dans l'optique d'un avantage métropolitain (Halbert, 2009) vis-à-vis des sites délocalisés.
\end{abstract}

Mots-clés: SEV, slalom, villes hôtes, conversion, métropolisation.

L'organisation des Jeux olympiques (JO) d'été amène à porter une réflexion sur le bâti, soulignant l'importance d'analyser la reconversion des équipements construits pour accueillir l'événement. Dans ce domaine, opérations urbaines et notoriété des territoires semblent en imbrication permanente. Si les méga-événements ont pu favoriser l'élaboration de stratégies d'aménagement touristique ou de valorisation territoriale, une âpre compétition s'est installée entre les métropoles. Alors que les élus sont de plus en plus contraints par la rationalisation des coûts, la mise en visibilité des anciens sites olympiques constituerait, pour eux, un instrument de valorisation territoriale. La reconversion touristique des sites apparaît dès lors comme un débouché possible pour accroître le rayonnement des métropoles hôtes. Cette affirmation demande toutefois à être vérifiée, tant cette croyance en l'héritage du bâti comme moyen de valoriser le territoire demeure tenace.

Pour en déconstruire les mécanismes, il convient de s'interroger sur l'impact de leur ouverture sur le territoire. L'objectif de cet article est de comprendre comment la reconversion touristique des sites olympiques délocalisés peut contribuer au développement métropolitain. Par sites délocalisés, j'entends l'ensemble des équipements conçus pour l'accueil des JO et éloignés géographiquement du village olympique, c'està-dire implantés en seconde couronne urbaine (Biard, 2012 : 14). Ces équipements spécialisés relèvent d'une architecture impropre à les intégrer au cour des cités et demandent une emprise au sol différente (Biard, 2012 : 14). Pour illustrer mes propos, je m'appuie sur des études de cas : l'aménagement des stades d'eau vive (SEV), rivières artificielles dédiées au slalom en canoë. Cette discipline, olympique depuis 1972, a été réintroduite à partir de 1992 grâce à des constructions reposant sur des investissements massifs pour l'accueil des JO (Endicott, 2007 : 26). En effet, l'activité nécessite la préexistence d'un bassin d'eau vive équipé de portes. Or, la ville choisie ne bénéficie pas toujours de rivières avec un dénivelé suffisant pour pouvoir aménager ce bassin (Marsac, 2008 : 89). Le SEV devient dès lors la seule alternative pour compenser une topographie peu propice aux aménagements de cours d'eau aux débits naturels (Goodman et Parr, 1994 : 195). 
Rappelons qu'à l'origine, le slalom constitue une discipline du canoë qui se déroule sur un courant rapide de la rivière. Pour utiliser les courants, les sportifs doivent franchir des portespiquets suspendues au-dessus de la surface de l'eau pour accentuer les difficultés techniques du torrent (Endicott, 2007 : 4). Si pour la plupart des kayakistes naviguer en eau vive c'est évoluer dans des espaces naturels, au XXI ${ }^{\mathrm{e}}$ siècle, plus de cinquante sites artificiels répertoriés ont été conçus à la fois pour accueillir des événements et participer à la mise en tourisme des territoires (Marsac, 2008 : 90). Depuis la fin des années 1990, dans la majorité des cas, le slalom se déroule dans ces espaces spécifiques en milieu urbain. Ce sont des aménagements où un fleuve est dévié et adapté pour la pratique du rafting et du kayak dans les villes. Des SEV se construisent, devenant des sites recevant des manifestations olympiques dont l'héritage demeure problématique et l'entretien, onéreux. En effet, l'édification de ces bassins artificiels représente un coût financier important pour la seule compétition olympique. Cela est dû au fait que depuis 2000, les débits des SEV sont exclusivement alimentés par des pompes électriques (Hydrostadium, 2014).

De plus, après les JO, le nombre d'utilisateurs sportifs demeure relativement faible : quelques milliers par ans (Boivin, 2005 : 34). Dès lors, la question de leur reconversion se pose en termes d'aménagement et d'offre touristique, car seules les nations investissant dans une réelle politique et possédant les fonds nécessaires peuvent choisir de subventionner de telles installations (les coûts engendrés par la mise aux normes des sites étant trop élevés). Les orientations d'aménagement s'imposent dès lors comme des leviers visibles de ces enjeux. Mais comment s'inscrivent-ils dans l'émergence de dynamiques territorialisées au sein des métropoles? Quelles stratégies les entités parties prenantes de la construction des SEV développent-elles pour la mise en tourisme des anciens sites olympiques?

Les JO constituent un événement majeur pour la discipline. En effet, ce n'est que tous les quatre ans que le canoë jouit d'une médiatisation d'ampleur mondiale (Endicott, 2007) comme en témoigne le succès des épreuves lors des trois dernières olympiades. Cette visibilité médiatique éphémère participerait de la notoriété des SEV. En m'attardant d'abord à expliciter le poids du méga-événement dans la construction des sites, je peux dresser l'inventaire des sites. Puis, j'exposerai les études de cas de quatre SEV : ceux d'Augsbourg, de Sydney, d'Athènes et de Londres. Je chercherai ensuite à saisir l'importance de l'équipement dans l'espace économique urbain, puis conclurai avec une discussion sur les effets de l'implantation du site dans la mise en tourisme du territoire, à travers le recours au concept d'«avantage métropolitain».

\section{Cadre conceptuel}

Parmi les travaux scientifiques consacrés à ce thème, les études se sont penchées sur ce phénomène de (re)conversion territoriale. La mise en œuvre de stratégies en faveur du tourisme et leurs répercussions sur l'urbanisation des métropoles a obligé les acteurs à reconsidérer le devenir des sites olympiques. Dans le sillage des géographes, la conversion des sites doit donc être reconsidérée à la lumière de l'évolution des métropoles.

Depuis les travaux de Jean-Pierre Augustin et de Pascal Gillon (2004), par delà les disciplines de référence, des chercheurs se sont interrogés sur le devenir des installations olympiques (Roult et Lefebvre, 2010; 2011 ; Bale et Mettekrogh, 2004; Gold et Gold, 2007), mais le processus de touristification des SEV olympique n'a pas encore été pris comme objet d'étude. Pour en comprendre les enjeux, il ne s'agit pas de se cantonner uniquement dans le registre de la compétition, mais de saisir comment les aménagements de sites olympiques associés aux conséquences en matière d'attraction pour les touristes ont pu constituer des leviers de développement métropolitain (Coulom et Bessy, 2014 : 77). Il convient de réfléchir en termes de métropolisation, processus qui renforce la dynamique et la capacité de développement du territoire. Ce phénomène résulte d'interactions entre acteurs agissant dans le cadre de leurs rôles sociaux qui restent complémentaires (c'est-à-dire les villes hôtes et l'État). La métropolisation du méga-événement peut être un processus qui détermine le fonctionnement des agglomérations (Roult et Lefebvre, 2011 : 40). Les approches possibles pour appréhender ce phénomène sont multiples. La métropolisation, au sens sociologique du terme, constitue un phénomène résultant de la concentration sur un territoire donné des fonctions et du potentiel de gestion, d'administration et de croissance économique comme résultat de la multiplication des interactions et des réseaux rattachant ce territoire aux autres entités métropolitaines.

Le concept d'«avantage métropolitain» (Halbert, 2009), emprunté à la géographie, interroge la capacité des villes à s'intégrer au sein d'un ensemble territorial plus vaste. Celui-ci rend compte de stratégies de mutualisation des moyens des villes formant la métropole, contribuant à son essor économique via le tourisme. Par exemple, le fait d'implanter le stade en périphérie urbaine permet ainsi d'élargir l'essor de ces infrastructures jusque-là supportées par la seule ville centre. Bien que le principe d' "avantage métropolitain» recouvre un ensemble hétérogène de pratiques et de représentations, il peut aussi bien désigner des retombées économiques ou l'impact positif en termes de notoriété de la métropole (Getz, 2008). Prenant la mesure de ces dimensions, les organisateurs de méga-événements s'interrogent sur le devenir des sites délocalisés. Les études menées sont destinées à trouver des solutions pour participer à la reconquête des marges de la métropole et rationaliser les coûts de gestion pour les parties prenantes (Boivin, 2005 : 56; Sylvestre, 2008).

De ce fait, la conversion touristique peut être lisible ici grâce aux opérations de reconquête des périphéries urbaines. Le marquage territorial par les SEV concerne toutes les entités métropolitaines, les espaces en déclin et non plus seulement les cœurs de ville (Roult et Lefebvre, 2011 : 38). Selon Augustin (2003: 51), ce «mouvement d'ex-urbanisation se renforce partout». La régiopolisation a un impact sur les villes hôtes dans lesquelles dominent les espaces polycentriques (Pflieger, 2013). De ce fait, les SEV s'implantent dans "l'aroundoor urbain" (Mao et al., 2013 : 90). Pour en comprendre les ressorts, il s'agit alors d'interroger ce développement des entités périphériques et de prendre en compte en priorité les SEV délocalisées à l'intérieur des frontières métropolitaines. En réalisant l'inventaire de ces sites, je vais d'une part, en révéler les caractéristiques puis, d'autre part, comprendre comment ils deviennent des pôles intégrés à la métropole. 


\section{Méthodologie \\ Les stades d'eau vive délocalisés : des lieux à convertir}

Pour présenter mes résultats, j'adopterai un plan chronologique qui permettra de restituer les étapes d'aménagement liées au SEV olympiques, des prémices d'une touristification des sites via leur implantation jusqu'aux processus présidant à leur conversion en sites touristiques.

\section{Genèse des sites olympiques délocalisés}

Dans le dernier tiers du $\mathrm{XX}^{\mathrm{e}}$ siècle, la question du legs des équipements olympiques est devenue une préoccupation majeure tant pour le développement des métropoles hôtes que dans la programmation de sites délocalisés. Les commissions du $\mathrm{CIO}$ ont remis en question, à plusieurs reprises, l'inscription du canoë de slalom comme discipline olympique au début du $\mathrm{XXI}^{\mathrm{e}}$ siècle (Projet de SEV de Rio, 2013). L'argument évoqué repose sur le coût des installations jugé onéreux par le $\mathrm{CIO}$ (archives CIO, 2014) et sur leur exploitation post-événement souvent perçue par les élus des villes hôtes comme peu rentable (Boivin, 2005 : 67). Des géographes rappellent que «la périodicité de ces méga-événements (tous les quatre ans) ou encore leur nombre limité au cours d'une année de championnat créent artificiellement un effet de rareté et une situation monopolistique pour les organisations qui en sont responsables» (Lefebvre et Roult, 2014 : 31). Il conviendra de revenir ici sur ce problème car il affecte fortement la propension des SEV à se reconvertir à moyen terme.

En effet, la construction d'un SEV s'inscrit dans des projets urbanistiques plus vastes : aménagements de friches, requalification de quartiers (Hydrostadium, 2014). Aujourd'hui, chaque SEV apparaît comme un site spécifique dédié au rafting (Boivin, $2005: 8$ ), les descentes du SEV représentant une extension touristique de la pratique du canoë.

Lorsque l'on étudie l'implantation des SEV, l'on pourrait croire, à l'instar des concepteurs et des élus des villes hôtes, qu'un lien prospère va automatiquement s'instaurer à l'échelle locale. Toutefois, le méga-événement olympique entraînerait des effets de halo qui participeraient à la croyance en un aménagement de sites pourvoyeurs de développement local (Roult et Lefebvre, 2011 : 32). Ce phénomène perdure dans les discours des parties prenantes et relève d'une construction sociale, car la délocalisation des sites engendre des coûts importants et il convient de revenir à leur phase d'élaboration pour comprendre comment des tensions ont accompagné l'attribution des sites aux villes candidates (Lunzenfichter, 2002). Cet auteur dévoile le rapport spécifique qui s'établit entre sport et État dans chacune des nations au cours de la période d'avant événement. J'identifie ces particularités en examinant les rapports officiels précédant l'attribution du premier bassin de compétition (à partir des Jeux de Munich de 1972).

\section{L'invention des sites délocalisés}

Bien que la question de la mise en tourisme du site n'ait pas encore été évoquée dans le débat public, en 1972, l'ancrage territorial faible de l'activité à cette période a incité le CIO à délocaliser le site de compétition hors de Munich. Le débat public est devenu un «enjeu de pouvoir pour les acteurs de l'aménagement» (Subra, 2014). La question de la (dé)localisation des sites se pose dès la construction de l'Eiskanal d'Augsbourg créé pour les JO de 1972. Les arguments évoqués par les élus pour bâtir un site éloigné du cœur de Métropole (Munich) reposent sur l'optimisation des infrastructures d'accueil et de transport. Ainsi, Willy Daume, président du Comité olympique allemand, déclare que «le parcours du canoë-slalom d'Augsbourg est situé à proximité immédiate du village Olympique. On y a installé une ligne de chemin de fer spéciale qui amènera les athlètes sur place en moins de 30 minutes à partir du village Olympique. Pratiquement, cette distance est plus faible que celle séparant le village Olympique du parcours de canoë-slalom munichois prévu à l'origine» (archives CIO, annexe XI : 12). Le comité d'organisation des JO décide en 1969 que dans l'optique où le CIO voterait pour l'inclusion du slalom dans la liste des disciplines olympiques, la ville d'Augsbourg accueillerait cette compétition (archives CIO, annexe VII : 25). L'Eiskanal, répondant à des normes de construction trop anciennes, est donc aménagé en adéquation avec les prérogatives de la discipline (archives CIO, annexe VII : 25). Depuis la fin des compétitions, ce site a été converti en parc boisé et la reconquête du site s'effectue par la baignade (CKM n ${ }^{\circ}$ 17, 1973 : 9). Le fait de délocaliser le site apparaîtrait comme les prémices d'une touristification, car des clubs implantés dans ce lieu accueillent des touristes pour les initier à l'activité (CKM n ${ }^{\circ} 75,1985$ : 11). Ce bassin a surtout servi de prototype pour les autres SEV olympiques (Boivin, $2005: 78$ ).

\section{La phase préolympique : une étape décisive}

Après 1972, le slalom disparaît pendant vingt ans, le coût des installations olympiques étant jugé trop important. La revue La Rivière titre "qu'il est impossible pour les pays pauvres de réaliser de tels équipements, mais le Canada en 1976 refuse, c'est un comble au pays d'origine du canoë!» (Bonnigal, 1973 : 4). Le déroulement de l'épreuve de slalom est remis en cause du fait de l'absence d'aménagements pour ce sport dans la ville de Montréal.

Le premier site délocalisé à exploiter le rafting comme activité touristique est le parc del Segre à Seo de Urgel. Bien que non programmée lors de la phase préolympique, sa mise en tourisme s'est effectuée par la vente de prestations d'encadrement de descentes en raft. Pourtant, dès l'année 1993, des emplois sont créés et l'équipement dynamise cette ville frontalière située à quinze kilomètres au sud d'Andorre (CKM $\mathrm{n}^{\circ} 109$ : 75). La période post-olympique a aussi permis d'amortir une partie du financement du SEV grâce à la production électrique d'une microcentrale intégrée au site (Ganyet, 2014). Cependant, le devenir du site n'est pas remis en question lors de la phase préolympique, contrairement au site d'Atlanta en 1996, l'Ocoee River, qui a été intégré dans un parc national et sert actuellement de base de rafting. Il a coûté « 24 millions de $\$$ (y compris le coût de l'événement) payés presque entièrement par l'État du Tennessee et l'Office National des forêts [...] 24000 personnes ont descendu l'Ocoee l'année dernière. En organisant des courses à tous niveaux [...] tout en répondant aux besoins touristiques, on compte bien ici rentabiliser le pari olympique dans les années à venir» (CKM n 128, 1995 : 3). 


\begin{tabular}{l|c|c|c}
\multicolumn{4}{c}{ Tableau 1: Types de site à partir des distances géographiques } \\
des SEV au village olympique
\end{tabular}

Si la rentabilité économique compte dans le choix de territorialisation de l'équipement, il convient de retenir aussi trois indicateurs géographiques pour comprendre le contexte dans lequel s'implantent les SEV. Il s'agit de restituer le nom de la localité dans laquelle est implanté le SEV, le type de territoire et la distance au village olympique. Pour les aménageurs, ces indicateurs permettent de répondre à l'équation du choix d'implantation des sites, comme le montre le tableau suivant.

La spécificité de ces sites de compétition liée à une importante emprise foncière conduit souvent à délocaliser les SEV aux frontières de la métropole dans les banlieues (Athènes, Londres, Sydney) quand ils ne sont pas implantés à plusieurs dizaines de kilomètres (Munich, Barcelone, Atlanta). Le tableau montre que ces SEV sont situés à au moins 20 kilomètres de la ville hôte, ce qui est en deçà de la moyenne de distance entre la ville centre et le village olympique. Les SEV se situent loin des centres-villes, à une distance moyenne de 56,42 kilomètres, dans l'ultra-périphérie des métropoles (tableau 1). On peut formuler l'hypothèse que cette distance moyenne au village olympique engendrerait des disparités dans la mise en tourisme des SEV délocalisés. Plus le site est proche de la ville centre, plus la fréquentation de ces équipements par les touristes deviendrait prégnante.

\section{Les conversions touristiques : réussites et limites}

Je vais désormais porter mon attention sur les cas de Penrith et Lee Valley qui relèvent d'un ancrage touristique au sein de la métropole. La comparaison est possible car ces villes se caractérisent par un habitat périphérique (grande banlieue, ville au sein d'un parc naturel urbain). Le SEV renforcerait l'offre touristique de ces entités spatiales des métropoles.

\section{Sydney : une conversion touristique rentable}

Le SEV de Penrith, ville située à 60 kilomètres à l'ouest de Sydney, a été, durant l'été 2000, le théâtre des épreuves olympiques tant en slalom qu'en course en ligne, ainsi qu'en aviron. Il y a une mutualisation des moyens qui a permis de réaliser des économies d'échelles (Billard, 2006 : 108). Grâce aux obstacles mobiles, le site de Penrith permet aussi bien l'organisation de compétitions de haut niveau qu'une utilisation ludique pour assurer la rentabilité de l'équipement. Cette cité australienne a été réaménagée en partie suite à l'ouverture au public de l'ancien équipement olympique. En effet, le SEV de Penrith est devenu le produit phare de l'offre touristique de la localité. Une politique en faveur du tourisme a offert la possibilité de faire évoluer l'image de cette ville grâce à une conversion territoriale sans précédent. Depuis la fin des JO, les gestionnaires réalisent un "chiffre d'affaires mensuel de plus de 100000 dollars australiens, ce qui lui permet d'afficher un bénéfice et des frais de fonctionnement du quart de cette somme» (Hydrostadium, 2014).

Depuis sa mise en eau en 1999, les SEV sont conçus par Hydrostadium, marque créatrice de ces sites et filiale d'Électricité en France. Le SEV de Penrith est devenu la référence en termes d'intégration dans la métropole du grand Sydney, car c'est la première fois que la reconversion d'un SEV a été planifiée avant l'événement (Vignais, 2002 : 55). En effet, le site devient rentable dès 1999 (Hydrostadium, 2014). Si le site répond à l'euphorie techniciste des JO, stimulé par le développement du loisir, ce bassin a dépassé la simple fonction d'arène de compétition pour se muer en un véritable complexe multimodal fréquenté par des milliers de touristes (Archives CIO, 2014). Plusieurs raisons expliquent cette concentration des citadins sur le site : la concomitance d'une quête du plaisir immédiat et d'une sécurité optimale en complément d'un matériel adapté (Hydrostadium, 2014). L'accès est d'abord facilité par des infrastructures routières et de transports (Roult et Lefebvre, 2011 : 37) érigées selon un modèle d'intégration du site à la ville hôte dit en «jointure» (Liao et Pitts, 2006) qui rentabilise les investissements initiaux consentis pour permettre la régénération et l'accessibilité des territoires visés par ces aménagements. Le cas de Londres s'en démarque car il est plus récent.

\section{Le SEV du Grand Londres :}

\section{les effets de la territorialisation}

Il faut maintenant examiner les effets locaux de territorialisation du SEV londonien. L'agencement du SEV de Londres s'est opéré pour les JO de 2012, faisant partie du Parc régional de Lee Valley dans l'Hertfordshire qui s'étend sur près de 42 kilomètres de long et couvre plus de 24000 hectares. Cet aménagement s'inscrit dans le London Plan de 2008 (Drozdz, 2014 : 132), politique redistributive des grands projets urbains visant à réaménager les périphéries de la capitale britannique. L'offre d'hébergement et de services périphériques à 45 minutes du centre de Londres entre dans un nouveau mode de gouvernabilité territoriale et peut être un outil au service des prestataires de tourisme car il recentre l'attractivité du SEV.

L'équipement a été mis en service fin 2010, soit plus de deux ans avant le début des JO, constituant un premier héritage olympique pour la métropole londonienne. Le SEV d'Eton sert actuellement d'équipement pour le canoë et le raft, et constitue un lieu d'entraînement et de compétition 
pour les athlètes d'élite. Le site est exploité par la «Lee Valley Regional Park Authority». L'aménagement a débuté en juillet 2009 (Cody, 2010 : 32). Le site est maintenant géré de manière autonome car il a été ouvert aux touristes. C'est le seul nouveau site du projet olympique de Londres 2012 qui a pu être utilisé par le public plusieurs mois avant les JO, puis reconverti en zone touristique avec hébergement. Les aménageurs ont devancé les organisateurs dans le but de valoriser la Lee Valley. Intégrée dans la politique du Grand Londres, la Lee Valley est un territoire accessible depuis la City. L'offre de la Lee Valley a été complétée par l'extension de zones de détente et d'une voie ferrée. La coopération induite par la mise en tourisme de villes situées dans les lisières du Parc naturel a indubitablement amené des clients issus des structures commerciales (voyagistes, guides). Dans les circuits touristiques, Lee Valley peut courir le risque de devenir une ville étape (comme c'est actuellement le cas de nombreuses banlieues) sur la route des hauts lieux touristiques. Le positionnement économique dans l'optique de contribuer à l' «avantage métropolitain » apparaît dès lors comme une variable déterminante via la politique de commercialisation des produits.

De plus, au sein du Grand Londres, la construction du SEV et sa mise en tourisme précoce ont été controversées, car l'utilisation intempestive du béton entre en contradiction avec le cahier des charges du parc naturel de Lee Valley, rendant la conversion territoriale mitigée et mettant en doute l'efficacité de la gouvernance métropolitaine de la capitale éponyme (Nativel, 2011). La réquisition du Parc naturel en 2007, par le biais du «compulsory purchasing» (droit de préemption de la mairie), a abouti, selon Joel Cody, à l'émergence d'une esthétique urbaine "décontextualisée et artificiellement plaquée sur papier glacé» (Cody, 2010). Tandis que les SEV de Penrith et de Lee Valley demeurent relativement éloignés des centres-villes, la distance géographique s'avère peu limitative : elle influencerait l'ancrage dans le territoire périphérique et accentuerait la dimension structurante des équipements. Ce n'est en revanche pas le cas d'Athènes.

\section{Une non-conversion : Athènes 2004}

Les stratégies des acteurs (CIO, États, etc.) permettent-elles toujours d'adapter l'offre touristique des SEV? Pour pondérer l'intégration des SEV olympiques dans les projets de territoire, il convient de revenir chronologiquement sur des cas de sites pensés uniquement pour accueillir les JO et qui aujourd'hui sont fermés (Athènes et Beijing). Initialement prévu à Marathon, le SEV des JO de 2004 s'est trouvé finalement localisé sur le site de l'ancien aéroport d'Hellinikon. Ce SEV sort du commun comme une innovation, car il réhabilite un espace désaffecté. La reconversion de ce lieu en périphérie des monuments anciens devait participer à une stratégie de reconquête et de renouvellement urbain (CIO, 2014).

Le bassin alimenté par un lac artificiel créé à cette occasion était lui-même rempli par de l'eau de mer grâce à une canalisation en « 8 » et à une station de pompage située sur la côte (Hydrostadium, 2014), mais le site demeure aujourd'hui inexploité. En effet, la rentabilité devait être amenée par l'activité récréative et touristique en rafting. Or, dans une ville comme Athènes, très orientée vers le patrimoine antique, mais où les températures sont souvent élevées, ce site n'a aucun intérêt touristique majeur. Le fonctionnement électrique et l'entretien du système de pompes ont entraîné le démantèlement des installations (Boivin, 2005 : 29). L'amortissement financier du SEV ne permettait pas d'exploiter l'espace de compétition.

De plus, l'aménagement intérieur du bâtiment d'accueil et les travaux paysagers ont engendré des coûts d'entretien trop élevés (Boivin, 2005 : 29). À l'origine, l'implantation de l'équipement devait participer au projet d'unité urbaine de la capitale grecque. Toutefois, ces investissements dans un contexte de crise financière ont précipité son effondrement. La spéculation immobilière a entraîné la fermeture temporaire du site et, de ce fait, les berges n'ont pas été aménagées (Bernard, 2014) car le déficit lié aux investissements de construction ne pouvait être comblé.

\section{Les facteurs décisifs : normes et spécificités}

Mais comment les SEV se transforment-ils sous l'effet de ces contraintes? Avec la médiatisation des épreuves de slalom, la compétition cristallise ces enjeux d'aménagement et de mise aux normes propres au spectacle sportif (Marsac, 2010). Ainsi, des parties de sites sont aménagées en conséquence. Le SEV est soumis à un double défi en termes de fonctionnalité et de modularité : réduire les coûts tout en restant attractif. Il faut alors améliorer les infrastructures nécessaires à la mise en tourisme (stationnement à proximité immédiat du SEV), aménager des aires confortables pour embarquer et débarquer et optimiser des possibilités de régulation du débit. Les dispositifs multimodaux renforceraient leur touristification.

\section{Des exigences en termes d'aménagement}

Depuis les années 1990, les spécificités des SEV sont présentes dans les cahiers des charges des sites (Goodman et Parr, 1994 : 192). Bien que les aménagements restent marqués par la compétition qui impose des règles aux organisateurs pour recevoir des spectateurs, ceux-ci répondent également à l'ouverture au public (normes, capacité d'accueil, etc.). L'innovation est ici appréhendée dans le contexte de spécialisation des espaces vers la logique des stades, condition nécessaire à la tenue des méga-événements, puis lieu d'accueil du public en proposant des services pour les familles et ceux qui ne pratiquent pas. Ce changement de conception de l'activité produite par l'avènement des SEV de seconde génération engendre une innovation structurante (Vignais, 2002 : 274), car les dispositifs multimodaux prévoient une conversion pour accueillir tous les publics. Pour interpréter ce phénomène, on avancera que l'innovation réside dans la conception d'espaces polyvalents et amorce une «mise aux normes» des installations. En établissant un dispositif spatial, les porteurs de projets recherchent la domestication du cours d'eau, dans la perspective d'améliorer l'accueil touristique. Il s'agit d'une phase d'innovation dans la mesure où différents profils de SEV se dégagent : certains ont des aménagements de débits quand d'autres, alimentés par des pompes électriques, adaptent leurs courants aux touristes novices (Hydrostadium, 2014). Mais cette accessibilité accrue entraîne des tensions générées par la nouveauté face aux normes instituées nécessaires à l'organisation du SEV (les modes d'accès des usagers aux infrastructures). J'ai identifié 


\begin{tabular}{l|l}
\hline \multicolumn{2}{c}{$\begin{array}{c}\text { Tableau 2: Les cinq phases de la conversion touristique } \\
\text { des SEV olympiques }\end{array}$} \\
\hline 1) Phase pré-événement & $\begin{array}{l}\text { Identification (ou non) du potentiel } \\
\text { touristique du SEV }\end{array}$ \\
\hline 2) Phase «événement» & $\begin{array}{l}\text { Diffusion territoriale et communication } \\
\text { du ClO }\end{array}$ \\
\hline 3) Phase post-événement & $\begin{array}{l}\text { Innovation et diversification de services } \\
\text { liées aux produits touristiques (rafting) }\end{array}$ \\
\hline 4) Moyen terme & $\begin{array}{l}\text { Segmentation de la clientèle et tensions } \\
\text { liées à l'accessibilité au SEV (touristes } \\
\text { vs sportifs) }\end{array}$ \\
\hline 5) Long terme & $\begin{array}{l}\text { Territorialisation du SEV, marketing } \\
\text { territorial et « avantage métropolitain » }\end{array}$ \\
\hline Source : Marsac, 2008. &
\end{tabular}

cinq étapes distinctes relevant de la conversion touristique des sites répertoriés dans le tableau ci-dessus.

La mise en tourisme des SEV olympiques se comprend donc à l'articulation de quatre facteurs : l'intégration au sein des métropoles hôtes, l'innovation dans l'offre de produits touristiques, le développement de services périphériques et le marketing territorial. Enfin, le processus de territorialisation des sites olympiques induit une adaptation à la mise en tourisme grâce à une mutualisation des moyens propres à «l'avantage métropolitain». La gestion des SEV est généralement conditionnée par des accords entre propriétaires, gestionnaires du site de pratique, usagers et collectivités locales.

$\mathrm{Du}$ fait des risques d'éviction, la conversion touristique nécessite une adaptation au marketing territorial, car selon les élus, avec les SEV, l'image de la ville hôte s'en trouverait améliorée (archives du CIO, 2014). Mais cette mise en tourisme, autour de laquelle la ville d'implantation du SEV communique, est-elle incluse dans une politique touristique visant «l'avantage métropolitain »? Ne profite-t-elle pas avant tout au centre de la métropole? Le cas du SEV d'Athènes qui a peiné à devenir un attrait touristique à cause de l'offre globale de la métropole davantage tournée vers le patrimoine amène à se questionner sur la fragilité de ce projet.

\section{Une pérennisation des sites par le tourisme?}

Le risque d'éviction du slalom du programme olympique représente une limite aux stratégies de conversion territoriale, car elle engendre, comme pour l'olympiade de Rio 2016, une frilosité de la part des élus dans l'investissement pour construire un nouveau SEV (projet de SEV de Rio, 2013). La dimension structurante de l'équipement repose sur sa notoriété liée à la participation internationale, le CIO demandant au moins 75 nations. En 2000, lors des JO de Sydney, 44 pays sont représentés, mais le problème majeur demeure la pérennisation des sites. Le projet de Sydney 2000 a failli ne jamais voir le jour. Pourtant, le fait d'abandonner ne dépend pas de la ville organisatrice, mais des facteurs de reconversion, en particulier conditionnés par le programme de développement de cette discipline et des usages de ces sites par des gestionnaires privés (contrat en délégation de service public, consortium). Là encore, le tourisme joue un rôle structurant (Augustin, 2008 : 41) même si le coût de gestion des sites freine les investisseurs (Boivin, $2005: 12$ ).

Aujourd'hui, c'est bien la valorisation des installations touristiques qui prime dans la décision de construire ou non des espaces dédiés à l'événement. Dans le cadre de l'avantage métropolitain, cette création d'infrastructures est conditionnée par la fonctionnalité et l'entretien des SEV par l'ensemble des parties prenantes. La question du devenir de ces installations se pose avec acuité pour tous les acteurs, car il s'agit d'exploiter le site de manière à rentabiliser les investissements consentis en participant collectivement aux charges d'entretien.

\section{Rentabiliser l'équipement dans le territoire périphérique}

À Athènes, les coûts de fonctionnement demeurent plus élevés qu'à Seo de Urgel (Hydrostadium, 2014). Si l'on ne peut anticiper sur le devenir touristique du site, peu de SEV sont aujourd'hui rentables dans leur globalité. Le coût de construction est trop élevé pour permettre d'englober l'amortissement de la construction dans les bilans (Boivin, 2005 : 9).

Les usages des SEV impliquent notamment des arbitrages financiers, car l'essence de ce sport lié à la nature repose désormais sur l'image des JO comme référent universel et «produit d'appel». Hors saison, les SEV créent une importante attractivité touristique comme en témoignent les cas de Penrith et Lee Valley (Marsac, 2014). Le fait de fréquenter un équipement périphérique modifie le rapport au temps et à l'espace : usages particuliers (saisons, moments privilégiés, durée, etc.) et symboliques (ordinaire/extraordinaire, vacances/séjour court) du temps de pratique; usages et sens symboliques des territoires (centre-ville/périphérie, local/non local, etc.), des lieux (de rassemblement, etc.) et des déplacements (urbain/périurbain, ville/autres villes, etc.). Le SEV perd donc son caractère « d'espace à la marge » pour se fondre dans la cité du XXI siècle.

Mais la transformation du SEV en lieu touristique a eu pour corollaire l'obligation à long terme, pour les usagers, de partager l'espace entre touristes et sportifs. À Lee Valley, un parcours destiné au tourisme et un autre à la compétition sont mis en fonction simultanément. Cela permet aux gestionnaires de concilier l'accueil des touristes avec les exigences du haut niveau. Pourtant, aujourd'hui, le site cherche encore sa cohérence territoriale même s'il participe aussi à «l'avantage métropolitain ». La politique de délocalisation du site s'est traduite par une extension plus que par un vrai renouvellement urbain. La connaissance du site par le grand public lui confere de nombreux atouts, car pour le bassin de vie de la métropole du Grand Londres, un consortium regroupe les autorités administratives et les gestionnaires de sites. "L'avantage métropolitain » doit être relativisé pour comprendre si le SEV permet de développer réellement le territoire. Les stratégies des élus participeraient de la mise en valeur des héritages sportifs, mais seraient peu reliées à l'habitation. Toutefois, la mise en tourisme des sites olympiques ne peut s'opérer sans une politique commerciale autour de produits phares (rafting). 


\section{La commercialisation de produits touristiques dans les SEV}

Dans la conversion territoriale, on assiste à la commercialisation de produits touristiques liés à la notoriété de services périphériques (hôtels, cafétéria sur place, clubs, etc.). Le phénomène marquant demeure la forte excroissance de l'offre à la périphérie de la métropole caractérisée par une marchandisation accrue liée à la notoriété olympique : «les JO s'insérant désormais dans des logiques de plus en plus marchandes et orientées autour de la notion d'image et de prestige de la ville hôte, ils s'incluent dans le giron moderne de la culture comme élément structurant des villes (Zukin, 1995 : 45), au sens où l'utilisation et la présence de celle-ci contribuent au développement et à l'affirmation de certains territoires » (Roult et Lefebvre, $2010: 369$ ).

Dans les SEV, si la prégnance du modèle urbain monocentrique a fait fuir les touristes, ces derniers séjournent désormais dans les périphéries (Lee valley, 2014). Dès lors, pour les élus, il s'agit de conjuguer innovation et rentabilité. La mise en tourisme des installations olympiques conduit souvent à des équilibres ou à des compromis précaires entre acteurs, comme en témoigne l'exemple des SEV conçus depuis la fin des années 1990. Athènes et Beijing ont démonté leurs sites. Bien que financés par l'État, ces équipements n'ont pas pu être rentabilisés, car ils ont été peu subventionnés lors de leur phase de mise en tourisme (Bernard, 2014) d'une part, et d'autre part, ils ont peu bénéficié de réelles stratégies marchandes sous l'égide des gestionnaires. Ces sites ont été fermés au public après les $\mathrm{JO}$ et sont alors devenus des friches.

Si le SEV réussit à être converti, il peut devenir un outil de marketing territorial. En effet, en utilisant le concept comme procédé de promotion du territoire, les collectivités territoriales cherchent à relancer le projet de développement local, mais le calcul d'impact économique des méga-événements sportifs sur les territoires repose sur une méthode ne prenant pas en compte l'ensemble des échanges avec les parties prenantes (Walo et al., 1996), notamment concernant les coûts d'opportunité (renoncement à des dépenses dans d'autres domaines).

Toutefois, les élus y croient car ils envisagent la mise en tourisme des sites comme une valeur ajoutée du territoire. Les offices de tourisme des villes périphériques des métropoles cherchent à asseoir leur identification touristique (Lee Valley, 2014), et à moyen terme, le marketing territorial relevant de la communication. Les stratégies de mise en tourisme des SEV participent à l'«avantage métropolitain» via les processus de marchandisation, mais elles peuvent être concurrencées par des équipements olympiques de prestige reconvertis (parcours d'équitation, vélodromes, salles spécialisées...). Les atouts des SEV se cristallisent autour de l'aspect récréatif du site dont la renommée rejaillit sur ce territoire quand les enceintes concurrentes jouissent d'une tradition plus ancienne et d'un positionnement mieux identifiable leur procurant un ancrage symbolique supérieur en même temps qu'une marque plus facilement commercialisable.

\section{Conclusion}

Les SEV en tant qu'équipements spécifiques renforcent la coopération métropolitaine bien que cette diversité des acteurs en présence, aux intérêts divergents, suscite des tensions. Si la position géographique de l'équipement sportif joue un rôle central dans la mise en tourisme des territoires, les villes dans lesquelles ces sites sont implantés profitent inégalement des retombées des JO. En effet, l'organisation de ce méga-événement nécessite d'aménager des infrastructures de manière à assurer leur desserte au sein de la métropole. Les SEV ancrés aux confins du territoire complètent l'offre existante dans la ville centre et ses banlieues. Ces équipements délocalisés participent à la diversification des activités propres aux métropoles dans lesquelles ils s'implantent, contribuant à «l'avantage métropolitain». Bien que concurrencés, ces sites commercialisés ne constituent pas seulement des revenus directs pour les territoires qui les hébergent, ils augmentent davantage la renommée du lieu et par là même, celle des métropoles. Les touristes, pratiquants occasionnels, représentent une cible pour le marketing territorial, segment qu'il faut capter pour rentabiliser le site. On assiste à une mutation de l'équipement au profit d'un espace multifonctionnel dédié en particulier au rafting dans l' "aroundoor urbain».

Cependant, la mise en tourisme des SEV présente des résultats contrastés comme l'illustre le démantèlement de sites. Comme pour le ski, le canoë «devient une pratique de masse sollicitée par l'industrie touristique, qui peu à peu l'éloigne de ses origines montagneuses» (Pivato, 1994 : 144). L'hypothèse d'une disparité dans la reconversion des sites peut-être validée car le devenir des sites est très variable selon les olympiades. Les SEV conçus à partir de 1999 ont réussi à se mettre en tourisme, car ils possèdent des atouts (multifonctionnels, accessibles aux novices). Si leur aménagement est resté pendant longtemps l'apanage des acteurs sportifs, la survivance des installations olympiques rencontre la commercialisation des activités touristiques comme gage de développement des périphéries. Le retour sur investissement lié à la notion de marketing territorial pour la destination touristique représenterait dès lors une dimension à ne pas négliger dans la compétition que se livrent les métropoles candidates à l'organisation des JO.

\section{Remerciements}

L'auteur tient à remercier les organismes suivants pour le financement de cette recherche : Centre d'études olympiques français (CEOF) et Académie nationale olympique française (ANOF) — programme de bourses 2014 .

\section{Sources}

Archives CIO - Comité international olympique (1970), Lausanne, Annexe VII, 25 p.

Archives CIO - Comité international olympique (1972), Lausanne, Annexe $\mathrm{XI}, 26 \mathrm{p}$.

Archives CIO — Comité international olympique (2014).

BERNARD, Gilles (2014) créateur de stades d'eau vive, entretien avec l'auteur, Pau : 18 septembre.

CKM - Canoë kayak magazine (1973), nº 17.

CKM - Canoë kayak magazine (1985), nº 75.

CKM - Canoë kayak magazine (année), nº 109.

CKM - Canoë kayak magazine (1995), nº 128.

GANYET, Francesc (2014) directeur du Parc olympique de Seo de Urgel, entretien avec l'auteur, Pau : 18 septembre. 


\section{Références}

AUGUSTIN, Jean-Pierre (2003) «L'importance de la culture sportive en milieu urbain», dans Sylvain LEFEBVRE (sous la direction de), Sports et villes - enjeux socio-économiques et socioculturels, Québec : Presses de l'Université du Québec, p. 43-57.

AUGUSTIN, Jean-Pierre (2008) «Pékin : après les JO, quel avenir pour les installations sportives?», Urbanisme, $\mathrm{n}^{\circ} 363$, novembre-décembre, p. 39-42.

AUGUSTIN, Jean-Pierre et Pascal GILLON (2004) L'Olympisme, bilan et enjeux géopolitiques, Paris : Armand Colin, $173 \mathrm{p}$.

BALE, John et Mitte KROGH CHRISTENSEN (2004) Post-olympism? Questioning sport in the twenty-first-century, Oxford : Berg. 230 p.

BERNARD, Gilles (2014) «Hydrostadium », <www.hydrostadium.com>, (consulté le 18 août 2014).

BIARD, Éric (2012) Facteurs de diffusion des pratiques sportives hors cadre et stratégie des acteurs : Études de cas comparatives dans la délocalisation des sports de nature aux milieux urbains, thèse de doctorat, Université de Paris Sud, $388 \mathrm{p}$.

BILLARD, Gérald (2006) «Après la compétition olympique, le village urbain durable. L'apport des Jeux d'été de 2000 à Sydney, Australie», Annales de la recherche urbaine, $\mathrm{n}^{\circ}$ 101, novembre, p. 101-107.

BOIVIN, Jean-Christophe (2005) Quels freins des collectivités territoriales à l'investissement dans un stade d'eau vive?, mémoire de maîtrise, management du sport, Université de Bourgogne, 129 p.

BONNIGAL, Daniel (1973) «Le bon et le mauvais. Réflexions de rentrée», La Rivière, $\mathrm{n}^{\circ} 453$, p. 4.

CODY, Joel (2010) «The Impossible dream: The London 2012 Olympics as a site for the projection of decontextualised futures", dans Deepa NAIK et Trenton OLDFIELD (sous la direction de), Critical Cities: Ideas, Knowledge and Agitation from Emerging Urbanists, vol. 2, London : Myrdle Court Press, p. 129-145.

COULOM, Jean-Christophe et Olivier BESSY (2014) «Du Stade d'Eaux Vives Pau-Pyrénées au Parc Naturel Urbain : Entre récréativité innovante et innovation territoriale», Loisir et société, vol. 37, n 2, avril, p. 79-100.

DROZDZ, Martine (2014) «La construction territoriale de la compétition et de la redistribution à Londres : entre rééchelonnement (rescaling) de l'État et enclavement stratégique», dans Antoine LE BLANC et al. (sous la direction de), Métropoles en débat. (dé)constructions de la ville compétitive, Nanterre: Presses universitaires de Paris Ouest, p. 131-150.

ENDICOTT, William (2007) Slalom E-Book: History of Slalom, Lausanne : International Canoe Federation, $189 \mathrm{p}$.

GETZ, Donald (2008) «Event tourism: Definition, evolution, and research", Tourism Management, vol. 29, $\mathrm{n}^{\circ} 3$, juin, p. 403-428.

GOLD, John et Margaret GOLD (sous la direction de) (2007) Olympic Cities, City Agendas, Planning and the World's Games, 1896-2012, New York : Routledge, $348 \mathrm{p}$.

GOODMAN, Franz et Gabriel PARR (1994) «The design and construction of artificial white water canoeing courses", Proceedings of the ICE Municipal Engineer, vol. 103, n 4, décembre, p. 191-202.

HALBERT, Ludovic (2009) L'avantage métropolitain, Paris : Presses universitaires de France, $143 \mathrm{p}$.

LEE VALLEY WHITE WATER CENTRE (2014), Lee Valley, <www.visitleevalley.org.uk/go/whitewater/>, (consulté le 18 août 2014).

LEFEBVRE, Sylvain et Romain ROULT (2014) «Méga-événements pour villes émergentes dominantes», Urbanisme, n 393, été, p. 31-33.

LIAO, Hanwen et Adrian PITTS (2006) «A brief historical review of Olympic urbanization ", International journal of the history of sport, vol. 23, $\mathrm{n}^{\circ} 7$, octobre, p. 1232-1252.
LUNZENFICHTER, Alain (2002) Athènes... Pékin (1896-2008) choix épiques des villes olympiques, Anglet : Atlantica, $322 \mathrm{p}$.

MARSAC, Antoine (2008) Canoë-kayak, des torrents au Stade d'eau vive, Thèse de doctorat, Université de Paris-Ouest, $590 \mathrm{p}$.

MARSAC, Antoine (2010) «La mise en spectacle d'un sport olympique : le canoë de slalom », Esporte e sociedad, <www.uff.br/esportesociedade/pdf/ es1604.pdf>, (consulté le 18 août 2014).

MARSAC, Antoine (2012) «Accueil des épreuves olympiques de slalom en canoë-kayak et développement territorial», Revue européenne de management du sport, $\mathrm{n}^{\circ} 36$, décembre, p. 59-72.

MARSAC, Antoine (2014) Gouvernance territoriale et aménagements destinés aux épreuves de slalom en canoë : vers un développement de stades d'eau vive olympiques sécurisés?, Rapport de recherche remis au Centre d'études olympiques français (CEOF) et à l'Académie nationale olympique française (ANOF).

MAO, Pascal; Jean CORNELOUP et Philippe BOURDEAU (2013) «L'indoor, l'underdoor et l'aroundoor : les sports de nature envahissent la ville", dans Sylvain LEFEBVRE, Romain ROULT et Jean-Pierre AUGUSTIN (sous la direction de), Les nouvelles territorialités du sport dans la ville, Québec: Presses de l'Université du Québec, p. 81-98.

NATIVEL, Corinne (2011) «Mobilisations urbaines et espaces de résistance aux Jeux olympiques de Londres et de Vancouver ", Observatoire de la société britannique, vol. 11, <http://osb.revues.org/1255>, (consulté le 18 août 2014).

PFLIEGER, Géraldine (2013) «Création et partage de la valeur ajoutée métropolitaine», dans Christian LEFÈVRE; Roseau NATHALIE et Tommaso VITALE (sous la direction de), De la ville à la métropole, les défis de la gouvernance, Paris : L'œil d'or, p. 199-212.

PIVATO, Stefano (1994) Les enjeux du sport, Paris : Casterman, 157 p.

MACKAY, Duncan (2013) Projet de Stade d'eau vive de Rio, Dunsar Media Company Limited, <http://www.insidethegames.biz/olympics/summerolympics/2016/1015868>, (consulté le 18 août 2014).

ROULT, Romain et Sylvain LEFEBVRE (2010) «Reconversion des héritages olympiques et rénovation de l'espace urbain : le cas des stades olympiques», Géographie, économie, société, vol. 12, nº 4, octobre-décembre, p. 367-391.

ROULT, Romain et Sylvain LEFEBVRE (2011) «Régénération urbaine, jeux olympiques d'été et planification durable et écologique du legs olympique : le cas du site d'Homebush Bay de Sydney», Loisir et Société, vol. $34, \mathrm{n}^{\circ} 2$, p. 31-65.

SUBRA, Philippe (2014) Géopolitique de l'aménagement du territoire, Paris : Armand Colin, $351 \mathrm{p}$.

SYLVESTRE, Gabriel (2008) «The social impacts of mega-events: towards a framework», Esporte e sociedade, vol. 4, n ${ }^{\circ} 10,<$ www.uff.br/esportesociedade/>. (consulté le 18 septembre 2014).

VIGNAIS, Ludovic (2002) «L'innovation structurante des espaces eaux vives", dans Olivier BESSY et de Dieter HILLAIRET (sous la direction de) Les espaces sportifs innovants, vol. 1, Voiron : Presses universitaires du sport, p. 263-289.

WALO, Maree; Adrian BULL et Helen BREEN (1996) «Achieving economic benefits at local events: a case study of a local sport event», Festival Management \& Event Tourism, vol. 4, nº 1, p. 95-106.

YIN, Robert K. (2003) Study Research, Design and Method, New York : Sage Publications, $181 \mathrm{p}$.

ZUKIN, Sharon (1995) The Cultures of Cities, Malden; Oxford : Blackwell Publishing, $338 \mathrm{p}$. 